\title{
Spububaúde
}

\section{Impacto da cirurgia bariátrica no metabolismo do cálcio}

\author{
Impact of bariatric surgery on calcium metabolism
}

\author{
José Fábio Monteiro Cintra ${ }^{1 *} \bullet$, Camila Chiara Pereira de Oliveira $^{2} \bullet$, Maria Vaniele Rodrigues

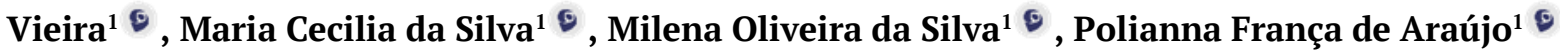 \\ ${ }^{1}$ Graduado em nutrição no Centro Universitário Unifavip-Wyden, Caruaru, Pernambuco, Brasil. ${ }^{2}$ Docente do Centro Universitário \\ Unifavip-Wyden, Caruaru, Pernambuco, Brasil. *Autor para correspondência: E-mail: fabiocintra05@gmail.com
}

\begin{abstract}
Resumo: Introdução: Devido à alta prevalência de obesidade que acomete o mundo inteiro, a cirurgia bariátrica vem ganhando força e espaço em relação aos tipos de tratamentos convencionais, principalmente por indivíduos que tem que perder peso, e que com apenas a dieta não vem obtendo sucesso. No entanto, esses procedimentos ocasionam diminuição importante na absorção de alguns nutrientes. Tendo como destaque, as concentrações séricas de cálcio. Dessa forma, foi realizada uma pesquisa bibliográfica com o objetivo de relatar as principais complicações causadas pela deficiência de cálcio em indivíduos submetidos à cirurgia bariátrica. Revisão e discussão: Utilizou-se a base de dados da BIREME para pesquisa dos artigos relacionados ao tema dos últimos 10 anos nos idiomas português e inglês. Foram incluídos ao final dez artigos, que demonstraram hipocalcemia em pacientes submetidos à cirurgia bariátrica, devido à baixa ingestão e absorção do cálcio, levando a alterações no paratormônio e ao aumento de maiores chances de desenvolver fraturas ósseas. Considerações finais: Pacientes submetidos à cirurgia bariátrica demostram diminuição nos níveis de cálcio, então se faz necessário averiguar de como manter esses níveis adequados para se evitar complicações provenientes dessa deficiência.
\end{abstract}

Palavras-chave: Cirurgia bariátrica, Cálcio, Hipocalcemia, Fraturas.

\begin{abstract}
Introduction: Due to the high prevalence of obesity that affects the whole world, bariatric surgery has been gaining strength and space in relation to the types of conventional treatments, mainly by individuals who have to lose weight, and who have not been successful with diet alone. However, these procedures cause a significant decrease in the absorption of some nutrients. Highlighting the serum calcium concentrations. Thus, a bibliographic research was carried out with the objective of reporting the main complications caused by calcium deficiency in individuals undergoing bariatric surgery. Review and discussion: The BIREME database was used to search for articles related to the theme of the past 10 years in Portuguese and English. At the end, ten articles were included, which demonstrated hypocalcemia in patients undergoing bariatric surgery, due to low calcium intake and absorption, leading to changes in parathyroid hormone and an increase in greater chances of developing bone fractures. Final remarks: Patients undergoing bariatric surgery show a decrease in calcium levels, so it is necessary to find out how to maintain these adequate levels.
\end{abstract}

Keywords: Bariatric surgery, Calcium, Hypocalcemia, Fractures.

\section{Introdução}

No Brasil, segundo o Ministério da Saúde, houve um aumento de 67,8\% no número de pessoas obesas entre 2006 e 2018 é o que retrata a Pesquisa de Vigilância de Fatores de Risco e Proteção para Doenças crônicas por Inquérito Telefônico (Vigitel), de 2018. Esse crescimento foi mais prevalente entre adultos de 25 a 34 anos, com $84,2 \%$. Já o excesso de peso acomete $55,7 \%$ da população, e a faixa etária de maior prevalência foi entre 18 e 24 anos. Quando levado em consideração o sexo, os homens apresentaram um aumento de $21,7 \%$, enquanto as mulheres $40 \%$ (Brasil, 2019).

Com a crescente incidência e prevalência de excesso de peso e obesidade no mundo, a cirurgia bariátrica vem se tornando uma alternativa frequentemente utilizada por pessoas que precisam perder peso e que já falharam nas alternativas convencionais. As cirurgias bariátricas se dividem basicamente em três procedimentos, tais como: Procedimento restritivo, disabsortivo e técnica mista (SBCBM, 2017).

O procedimento de técnica mista é o mais realizado no Brasil e no mundo, pois causa restrição no tamanho do estômago em receber alimentos, e um pequeno desvio no intestino provocando uma discreta má absorção de nutrientes. Vale ressaltar, que esse tipo de cirurgia é considerado como padrão ouro (SBCBM, 2017). 
No entanto, é importante destacar que esses indivíduos submetidos à cirurgia bariátrica, principalmente pelo método misto, apresentam maior risco de desenvolver deficiências nutricionais, pois há uma diminuição severa tanto na ingestão de alimentos, quanto na absorção de nutrientes (Krause, Mahan, \& Escott-Stump, 2010).

Deste modo, é evidenciado em alguns estudos que ocorre uma redução nas concentrações séricas de cálcio. Esse micronutriente desempenha muitas funções no nosso organismo, principalmente no metabolismo ósseo, que devido a sua má absorção após o procedimento cirúrgico, poderá apresentar hipocalcemia, alteração no hormônio paratormônio, bem como o surgimento de fraturas ósseas. Causando assim, alterações importantes na saúde do paciente bariátrico (Schafer et al., 2015; Lu et al., 2015; Yu et al., 2015; Costa et al., 2016; Shah et al., 2017; Melo et al., 2017; Wei et al., 2018). Considerando esses aspectos apontados, o objetivo dessa revisão é relatar as principais complicações causadas pela deficiência de cálcio nos pacientes submetidos à cirurgia bariátrica.

\section{Revisão}

Foi realizada uma pesquisa bibliográfica na base de dados BIREME, com os descritores na língua portuguesa e inglesa: cirurgia bariátrica "bariatric surgery"; cálcio "calcium"; fraturas "fractures"; osteoporose "osteoporosis" e tecido ósseo "bone tissue". Foram selecionados artigos originais relacionados ao tema dos últimos 10 anos.

Inicialmente a busca resultou em 395 trabalhos a partir do tema abordado, posteriormente foram selecionados 10 artigos de acordo com os seguintes critérios de inclusão: artigos com seres humanos, artigos originais publicados na língua inglesa e portuguesa. Em relação aos critérios de exclusão, foram excluídos às teses, dissertações, trabalhos de conclusão de curso, revisões de literatura e artigos com animais.

No quadro 1 apresenta as características dos dez artigos selecionados. Foram analisados os seguintes pontos: riscos de fraturas, concentrações séricas de cálcio e paratormônio (PTH), hipocalcemia e hiperparatiroidismo secundário (SHPT) entre pacientes submetidos à cirurgia bariátrica.

Dos dez estudos apreciados, sete foram de coortes; dois transversais e um caso-controle. Todos avaliaram os indivíduos por no mínimo 6 meses, e também foram analisadas as concentrações séricas de cálcio, o paratormônio e o risco de fraturas. Dos tipos de procedimentos bariátricos, o RYGB foi o mais prevalente, estando descrito em oito pesquisas.

Quadro 1. Categorização dos artigos utilizados no presente estudo quanto à amostra, tipo de estudo e cirurgia, tempo pós-cirurgia e os principais resultados.

\begin{tabular}{|c|c|c|c|c|}
\hline Autor/ano & $\begin{array}{c}\text { Amostra/tipo de } \\
\text { estudo }\end{array}$ & Tipo de cirurgia & $\begin{array}{l}\text { Tempo de } \\
\text { cirurgia }\end{array}$ & Resultados \\
\hline Schafer et al. (2015) & $\begin{array}{l}33 \text { adultos/ coorte } \\
\text { prospectiva. }\end{array}$ & RYGB & 6 meses. & $\begin{array}{l}\text { Foi verificado um declínio da FCA que } \\
\text { no pré-operatório era de } 32,7 \% \\
\text { passando a ser no pós-operatório de } \\
\text { 6,9\%. No entanto, os marcadores de } \\
\text { remodelação óssea aumentaram } \\
\text { acentuadamente e o PTH também } \\
\text { aumentou. } \\
\end{array}$ \\
\hline Shah et al. (2017) & $\begin{array}{c}999 \text { pacientes/ } \\
\text { coorte retrospectiva. }\end{array}$ & RYGB e BPS-DS. & 12 meses. & $\begin{array}{l}\text { A prevalência de hipocalcemia foi de } \\
1,9 \% \text { no grupo RYGB, } 9,3 \% \text { no grupo } \\
\text { gastrectomia vertical e } 10 \% \text { no grupo } \\
\text { BPD-DS. }\end{array}$ \\
\hline Costa et al. (2016) & $\begin{array}{l}83 \text { adultos/ } \\
\text { observacional, } \\
\text { transversal. }\end{array}$ & RYGB & $\begin{array}{l}\text { No mínimo } \\
12 \text { meses. }\end{array}$ & $\begin{array}{l}\text { Hiperparatiroidismo secundário foi } \\
\text { presente apena no OG. A densidade } \\
\text { mineral óssea foi significativamente } \\
\text { menor no OG. }\end{array}$ \\
\hline Lu et al. (2015) & $\begin{array}{l}2064 \text { adultos/ } \\
\text { coorte. }\end{array}$ & $\begin{array}{c}\text { Gastrectomia } \\
\text { laparoscópica, } \\
\text { aberta ou parcial e } \\
\text { RYGB. }\end{array}$ & 12 anos. & $\begin{array}{l}\text { No geral, houve um aumento } \\
\text { significativo no risco de } 1,21 \text { vezes de } \\
\text { fratura no grupo cirúrgico em } \\
\text { comparação com o grupo controle. }\end{array}$ \\
\hline Rousseau et al. (2016) & $\begin{array}{l}12676 \text { pacientes/ } \\
\text { caso-controle } \\
\text { retrospectivo } \\
\text { aninhado. }\end{array}$ & $\begin{array}{l}\text { BPS-DS; RYGB; } \\
\text { LSG e LAGB. }\end{array}$ & 8 anos. & $\begin{array}{l}\text { O grupo de cirurgia bariátrica tanto } \\
\text { antes quanto depois da cirurgia estava } \\
\text { mais propício a risco de fraturas em } \\
\text { comparação ao grupo controle. }\end{array}$ \\
\hline
\end{tabular}




\begin{tabular}{|c|c|c|c|c|}
\hline Yu et al. 2017 & $\begin{array}{c}15.032 \text { adultos/ } \\
\text { coorte. }\end{array}$ & RYGB e AGB. & $\begin{array}{c}\text { No mínimo 1 } \\
\text { ano. }\end{array}$ & $\begin{array}{c}\text { O RYGB está associado a um risco } \\
\text { aumentado de 43\% de fratura não } \\
\text { vertebral comparado com AGB. }\end{array}$ \\
\hline Yu et al. (2015) & $\begin{array}{c}\text { 30 adultos/ coorte } \\
\text { prospectivo. }\end{array}$ & RYGB & 24 meses. & $\begin{array}{c}\text { Aos 24 meses a DMO foi 5\% a 7\% mais } \\
\text { baixa na coluna vertebral e 6\% a 10\% } \\
\text { mais baixa no quadril em indivíduos } \\
\text { submetidos à cirurgia bariátrica em } \\
\text { comparação com os controles não } \\
\text { cirúrgicos. }\end{array}$ \\
\hline Wei et al. (2017) & $\begin{array}{c}1470 \text { pacientes/ } \\
\text { coorte retrospectiva. }\end{array}$ & SAGB, LAGB e LSG. & 12 meses. & $\begin{array}{c}\text { Aumento nos níveis de HPTS após } \\
\text { cirurgia. }\end{array}$ \\
\hline Melo et al. (2017) & $\begin{array}{c}91 \text { adultos/tipo } \\
\text { seccional, } \\
\text { transversal. }\end{array}$ & RYGB ou BPS-DS & $\begin{array}{c}\text { No mínimo 6 } \\
\text { meses. }\end{array}$ & $\begin{array}{c}\text { Não foram encontradas diferenças } \\
\text { significativas no PTH, mas os pacientes } \\
\text { cirúrgicos apresentaram aumento na } \\
\text { reabsorção óssea. }\end{array}$ \\
\hline Guglielm et al. (2017) & $\begin{array}{c}42 \text { indivíduos/ } \\
\text { coorte. }\end{array}$ & LSG & 6 meses. & $\begin{array}{c}\text { Redução do PTH após cirurgia e não } \\
\text { obteve diferença significativa entre } \\
\text { cálcio e DMO no pré e pós-operatório. }\end{array}$ \\
\hline
\end{tabular}

OG: grupo operado, PTH: paratormônio, DMO: densidade mineral óssea, FCA: absorção fracional intestinal de Ca, RYGB: Bypass gástrico em Y de Roux, BPS-DS: derivação biliopancreática por duodenal switch, HPTS: hiperparatireoidismo secundário, AGB: bandagem gástrica ajustável, SAGB: anastomose única (mini) cirurgia gástrica, LAGB: bandagem gástrica ajustável laparoscópica, LSG: gastrectomia vertical.

\section{Discussão}

De acordo com os estudos analisados a maioria demonstram alterações nas concentrações de cálcio em indivíduos submetidos à cirurgia bariátrica, mostrando que esse procedimento compromete a absorção do cálcio levando a hipocalcemia e consequentes problemas em sua metabolização (Schafer et al., 2015; Shah et al., 2017). Esses achados, foram verificados no estudo de Schafer et al. (2015) que mesmo com suplementação individualizada de cálcio, no pós-cirúrgico do RYGB, para se atingir $1200 \mathrm{mg} / \mathrm{dia}$ associada a suplementação adequada de vitamina $\mathrm{D}$, houve prevalência da diminuição da FCA e diminuição do cálcio absorvido.

Semelhante a pesquisa anterior, Shah et al. (2017) também observou que com a suplementação de cálcio de acordo com cada procedimento cirúrgico, para a RYGB foram administrados $940 \mathrm{mg} / \mathrm{dia}$, na gastrectomia $739 \mathrm{mg} /$ dia e $1612 \mathrm{mg} /$ dia no desvio biliopancreatico, adicionado a uma suplementação com vitamina D. Assim, foi observado que 3,6\% dos pacientes apresentaram hipocalcemia (cálcio sérico $\leqslant 8,9 \mathrm{mg} / \mathrm{dl}$ ), e o procedimento desvio biliopancreático foi o que apresentou maior percentual, cerca de $10 \%$.

Divergindo do que foi anteriormente mencionado, na pesquisa de Costa et al. (2016) foi evidenciado no pós-operatório da cirurgia bariátrica pela RYGB, que $67,8 \%$ dos indivíduos submetidos ao procedimento cirúrgico receberam suplementação de $188,02 \mathrm{mg} /$ dia de cálcio pelo período de 16,1 meses e apresentaram cálcio sérico normais. Isso pode ter ocorrido devido o tipo de cirurgia que foi RYGB, e também pela quantidade de cálcio suplementado que foi o mesmo para todos os indivíduos.

Em relação às fraturas, variante investigada nos estudos, foi verificado que são as principais preocupações nos pacientes bariátricos. Visto que, traz modificações importantes na qualidade de vida dos pacientes submetidos a esses procedimentos cirúrgicos. Isso ocorre, normalmente, por uma mobilização do cálcio dos ossos para a corrente sanguínea com intuito de manter a homeostase desse mineral, e que assim possa desempenhar seu papel no organismo (Lu et al., 2015; Rousseau., 2016; Yu et al., 2017).

Como foi observado na pesquisa de Lu et al. (2015) que evidenciou um aumento de 1,21 vezes de riscos de fraturas em pacientes submetidos a gastroplastia. A pesquisa também mostrou que entre 1 e 2 anos pós bariátrica é o período de maior tendência de riscos de fraturas. Da mesma forma, no estudo de (Rousseau., 2016) foi identificado que os pacientes apresentaram, claramente, riscos maiores de fraturas, quando submetidos principalmente ao procedimento cirúrgico de desvio biliopancreatico. Isso pode ser devido à técnica empregada nesse procedimento.

Já no estudo de Yu et al. (2017) evidenciou-se um total de 281 fraturas não vertebrais, sendo $43 \%$ maior no RYGB do que na banda gástrica ajustável. Isso deve ter ocorrido devido o RYGB ser um procedimento misto, e no caso do método por banda gástrica ajustável ser apenas um procedimento restritivo. Diferente desses resultados, na casuística de Yu et al. 2015 não foi verificada nenhuma fratura, mesmo com os marcadores de remodelação óssea aumentados e a DMO diminuída. Esse fato pode ser explicado devido ao reduzido tamanho amostral e tempo de seguimento.

Outra alteração, bastante prevalente, foi o aumento da secreção do PTH devido à presença de hipocalcemia (Costa et al., 2016; Wei et al., 2018). Esse desajuste foi encontrado na amostra de Costa et al. (2016) que além 
do PTH aumentado $(68,1 \mathrm{pg} / \mathrm{ml})$, evidenciou-se SHPT em quase 50\% desse grupo. O SHPT está relacionado com o PTH $>67 \mathrm{pg} / \mathrm{ml}$, vitamina D baixa $<30 \mathrm{mg} / \mathrm{ml}$ e valor de cálcio normal ou abaixo. Corroborando com esses achados, no que diz respeito ao SHPT, para (Wei et al., 2018) o SHPT estava presente em $21 \%$ dos indivíduos, antes da cirurgia bariátrica, e esse número aumentou para $37,2 \%$ no pós-operatório. Os procedimentos by-pass gástricos foram os que apresentaram maior aumento no pós-cirúrgico de SHPT. É importante destacar, que esse estudo foi realizado com asiáticos, e o aumento do SHPT após by-pass gástrico está atribuído ao comprometimento da absorção, e a uma ingestão reduzida de cálcio. Assim, pode ser justificado pela baixa ingestão de alimentos fonte de cálcio e baixa exposição solar por essa população.

Contudo, o estudo de Melo et al. (2017) difere dos demais, no que diz respeito ao PTH, pois ele não mostrou aumento desse hormônio nos indivíduos submetidos a cirurgia. Descreve também, que houve uma ingestão dietética de cálcio, porém abaixo da recomendação no pós-cirúrgico.

Em contrapartida, no pós-operatório de gastrectomia, foi observada uma diminuição do PTH, porém não teve mudanças nas concentrações do pré e pós-operatório de cálcio e vitamina $\mathrm{D}$, mesmo com suplementação. É importante frisar, que essa foi a única pesquisa da presente revisão, o qual mostrou redução do PTH após o procedimento. Essa contrariedade pode ser devido ao tipo de procedimento e o tamanho da amostra (Guglielm et al., 2018).

\section{Considerações finais}

Diante do que foi mencionado, compreende-se que os pacientes que são submetidos à cirurgia bariátrica mostram alterações no metabolismo do cálcio, e essas modificações levam a hipocalcemia comprometendo as funções desempenhadas pelo cálcio. Deste modo, ocasionando aumento do PTH e riscos de fraturas. Portanto, se faz necessário averiguar como manter o nível de cálcio adequado nos indivíduos submetidos à gastroplastia, seja por uma dieta rica em alimentos fontes de cálcio, ou por suplementação mais adequada e individualizada.

\section{Referências}

Brasil. 2019. Ministério da Saúde. Brasileiros atingem maior índice de obesidade nos últimos treze anos. Disponível em: https://www.saude.gov.br/noticias/agencia-saude/45612-brasileiros-atingem-maiorindice-de-obesidade-nos-ultimos-treze-anos. Acesso em: 26 mai. 2020.

Costa, T. M. D. R. L., Paganoto, M., Radominski, R. B., \& Borba, V. Z. C. 2016. Impacto da deficiência nutricional na massa óssea após cirurgia bariátrica. ABCD. Arquivos Brasileiros de Cirurgia Digestiva, 29(1), 38-42.

Guglielmi, V., Bellia, A., Gentileschi, P., Lombardo, M., D’Adamo, M., Lauro, D., \& Sbraccia, P. (2018). Parathyroid hormone in surgery-induced weight loss: no glucometabolic effects but potential adaptive response to skeletal loading. Endocrine, 59(2), 288-295.

Krause, M. V., Mahan, L. K., \& Escott-Stump, S. 2010. Krause: alimentos, nutrição e dietoterapia (12a ed.). Rio de Janeiro, RJ: Elsevier.

Lu, C. W., Chang, Y. K., Chang, H. H., Kuo, C. S., Huang, C. T., Hsu, C. C., \& Huang, K. C. (2015). Fracture risk after bariatric surgery: a 12-year nationwide cohort study. Medicine, 94(48), e2087.

Melo, T. L., Froeder, L., Baia, L. D. C., \& Heilberg, I. P. (2017). Bone turnover after bariatric surgery. Archives of endocrinology and metabolism, 61(4), 332-336.

Rousseau, C., Jean, S., Gamache, P., Lebel, S., Mac-Way, F., Biertho, L., ... \& Gagnon, C. (2016). Change in fracture risk and fracture pattern after bariatric surgery: nested case-control study. Bmj, 354, i3794.

Sociedade Brasileira de Cirurgia Bariátrica e Metabólica [SBCBM]. 2017. A Cirurgia Bariátrica. Disponível em: https://www.sbcbm.org.br/a-cirurgia-bariatrica/. Acesso em: 24 ago. 2019.

Schafer, A. L., Weaver, C. M., Black, D. M., Wheeler, A. L., Chang, H., Szefc, G. V., ... \& Shoback, D. M. (2015). Intestinal calcium absorption decreases dramatically after gastric bypass surgery despite optimization of vitamin D status. Journal of Bone and Mineral Research, 30(8), 1377-1385.

Shah, M., Sharma, A., Wermers, R. A., Kennel, K. A., Kellogg, T. A., \& Mundi, M. S. (2017). Hypocalcemia after bariatric surgery: prevalence and associated risk factors. Obesity Surgery, 27(11), 2905-2911.

Wei, J. H., Lee, W. J., Chong, K., Lee, Y. C., Chen, S. C., Huang, P. H., \& Lin, S. J. (2018). High incidence of secondary hyperparathyroidism in bariatric patients: comparing different procedures. Obesity Surgery, 28(3), 798-804. 
Yu, E. W., Bouxsein, M. L., Putman, M. S., Monis, E. L., Roy, A. E., Pratt, J. S., ... \& Finkelstein, J. S. (2015). Two-year changes in bone density after Roux-en-Y gastric bypass surgery. The Journal of Clinical Endocrinology \& Metabolism, 100(4), 1452-1459.

Yu, E. W., Lee, M. P., Landon, J. E., Lindeman, K. G., \& Kim, S. C. (2017). Fracture risk after bariatric surgery: Roux-en-Y gastric bypass versus adjustable gastric banding. Journal of Bone and Mineral Research, 32(6), $1229-1236$.

\section{Minicurrículo}

José Fábio Monteiro Cintra. Possui graduação em nutrição no Centro Universitário Unifavip Wyden (2019). Pós-Graduação em nutrição clínica no Centro Universitário Unifavip Wyden em andamento.

Camila Chiara Pereira de Oliveira. Possui graduação em nutrição pela Universidade Federal de Pernambuco (2009), especialização em nutrição Esportiva pela Gama Filho (2011), residência em nutrição clínica pelo programa de pós-graduação da HUOC/UPE (2013) e mestrado em Nutrição pela Universidade Federal de Pernambuco (2015). Atualmente professora do Centro Universitário UNIFAVIP.

Maria Vaniele Rodrigues Vieira. Possui graduação em nutrição no Centro Universitário Unifavip Wyden (2019).

Maria Cecilia da Silva. Possui graduação em nutrição no Centro Universitário Unifavip Wyden (2019).

Milena Oliveira da Silva. Possui graduação em nutrição no Centro Universitário Unifavip Wyden (2019). Pós-graduação em nutrição clínica, metabolismo, prática e terapia nutricional (FAVENI) em andamento.

Polianna França de Araújo. Possui graduação em nutrição no Centro Universitário Unifavip Wyden (2019). Pós-Graduação em nutrição clínica e funcional (INADES) em andamento e pós-graduação em nutrição clinica, metabolismo, prática e terapia nutricional (FAVENI) em andamento.

Como citar: Cintra, J.F.M., Oliveira, C.C.P., Vieira, M.V.R., Silva, M.C., Silva, M.O., \& Araújo, P.F. 2020. Impacto da cirurgia bariátrica no metabolismo do cálcio. Pubsaúde, 4, a070. DOI: https://dx.doi.org/10.31533/pubsaude4.a070

Recebido: 7 set. 2020 .

Revisado e aceito: 16 set. 2020.

Conflito de interesse: os autores declaram, em relação aos produtos e companhias descritos nesse artigo, não ter interesses associativos, comerciais, de propriedade ou financeiros que representem conflito de interesse.

Licenciamento: Este artigo é publicado na modalidade Acesso Aberto sob a licença Creative Commons Atribuição 4.0 (CC-BY 4.0). 